\title{
Numerical study of two-dimensional disordered Klein-Gordon lattices with cubic soft anharmonicity
}

\author{
J Cuevas ${ }^{a}$, JFR. Archilla ${ }^{b}$, F Palmero $^{b}$ and FR Romero ${ }^{a 1}$ \\ a Faculty of Physics, Avda. Reina Mercedes,41012-Sevilla, Spain \\ ${ }^{b}$ Faculty of Computer Science, Avda Reina Mercedes,41012-Sevilla, Spain \\ E-mail: ${ }^{1}$ romero@cica.es
}

\begin{abstract}
.
Localized oscillations appear both in ordered nonlinear lattices (breathers) and in disordered linear lattices (Anderson modes). Numerical studies on a class of two-dimensional systems of the Klein-Gordon type, show that there exist two different types of bifurcations in the path from nonlinearity-order to linearitydisorder: inverse pitchforks, with or without period doubling, and saddle-nodes. This was discovered for a one-dimensional system in a previous work of Archilla, MacKay and Marin. The appearance of a saddle-node bifurcation indicates that nonlinearity and disorder begin to interfere destructively and localization is not possible. On the contrary, the appearance of a pitchfork bifurcation indicates that localization persists.
\end{abstract}

PACS numbers: 63.20.Pw, 63.20.Ry

Submitted to: J. Phys. A: Math. Gen.

\section{Introduction}

Some models of spatially discrete systems consider particles with anharmonic potentials and, usually, the distribution of masses and couplings are supposed spatially homogeneous (nonlinear ordered systems). Other models refer to particles with harmonic interactions and a random distribution of masses and couplings (linear disordered systems).

Time-periodic and spatially-localized oscillations (LOs) appear in these dynamical systems due to different causes: (i) in nonlinear ordered systems of weakly coupled oscillators, LOs are caused by nonlinearity and they are called breathers $[1,2,3]$; (ii) in linear disordered systems, LOs are a consequence of disorder, and they are referred as Anderson modes [4]. In nonlinear-disordered systems, localization can be produced by any of the two causes. That is why, in this paper, we refer to them as LOs.

For a system with both nonlinearity and disorder, it is of great importance to know which is the dominant mechanism producing localization. This problem has been object of recent research [5, 6, 7], and different approaches has been proposed.

One approach considers systems with fixed degrees of nonlinearity and disorder, and LOs are studied varying the breather frequency $[6,7]$. Another approach considers 
a class of systems such that both nonlinearity and disorder can be varied continuously from the nonlinear ordered limit to the linear disordered limit. By means of the study of a class of one-dimensional models of the Klein-Gordon type, it has been proved numerically [5] that breathers can be continuously connected to Anderson modes.

The studies developed in disordered anharmonic media has been carried out in one-dimensional systems. The next step could be to address the problem in twodimensional systems, which have been widely studied without disorder [8, 9, 10]. These models can be used to explain some properties of layers in solids [11, 12], hightemperature superconductors [13] and photonic crystal waveguides [14]. Thus, it can be interesting to address the question whether in these two-dimensional systems there also exists a continuous path that transforms breathers into Anderson modes. This study would allow to clarify the interplay between the two mechanisms of localization in two-dimensional lattices.

In this paper, we have found an affirmative answer to the question about the existence of a connection of some families of discrete breathers to Anderson modes, in two-dimensional lattices.

\section{The model}

We study an anharmonic Hamiltonian square lattice of the Klein-Gordon type, i.e., with an on site potential. The Hamiltonian is:

$$
\begin{aligned}
& H=\sum_{n_{1} n_{2}=1}^{m} \frac{1}{2} p_{n_{1} n_{2}}^{2}+\frac{1}{2} \omega_{n_{1} n_{2}}^{2} u_{n_{1} n_{2}}^{2}-s u_{n_{1} n_{2}}^{3}+ \\
& +\frac{1}{2} \varepsilon \sum_{\left|n_{1}-n_{1}^{\prime}\right|=1} \sum_{\left|n_{2}-n_{2}^{\prime}\right|=1}\left(u_{n_{1} n_{2}}-u_{n_{1}^{\prime} n_{2}^{\prime}}\right)^{2},
\end{aligned}
$$

where $u_{n_{1} n_{2}}$ are the coordinates of the particles with respect to their equilibrium positions; $\omega_{n_{1} n_{2}}$ are the frequencies of the small amplitude oscillation of the particles; $-s u_{n_{1} n_{2}}^{3}$ is the anharmonic part of the on-site potential for the particle $\left(n_{1}, n_{2}\right) ; s$ is a parameter which describes the degree of anharmonicity and takes its value in $[0,1]$, being $s=0$ the harmonic-disordered case, and $s=1$, the anharmonic-ordered case; and $\varepsilon$ is a coupling parameter. The coupling potential is harmonic and nearest neighbour, though both this assumptions can be relaxed.

The disorder is implemented by means of the curvatures $\omega_{n_{1} n_{2}}^{2}$ of the local potentials at their minima. Since the masses have all been chosen equal to 1 , this gives rise to frequencies $\omega_{n_{1} n_{2}}$. Suppose they take two values randomly distributed, say:

$$
\omega_{n_{1} n_{2}}=\omega_{0}\left(1+\rho \frac{r_{n_{1} n_{2}}}{2}\right)
$$

where $\rho$ is a disorder parameter that takes its value in the interval $[0,1]$ and $r_{n_{1} n_{2}}$ are the components of a random vector which can be either +1 or -1 . At $\rho=0$, there is no disorder and all the frequencies are equal to $\omega_{0}$, which is taken with value 1 . At $\rho=1$, the most disordered case, the frequencies are either $0.5 \omega_{0}$ or $1.5 \omega_{0}$. This is the most important difference between our model and the one studied by Kopidakis and Aubry [6] since they take an continuous distribution of random frequencies. 
The parameter $\rho$ is considered a monotonic function $\rho=\rho(s)$ of the nonlinearity $s$, with $\rho(0)=1$ and $\rho(1)=0$, giving a path from the anharmonic ordered case to the harmonic disordered case. The paths used in this paper are of the type:

$$
\rho(s)=1-s^{q}, \quad q>0,
$$

and specifically we have used the values $q=1 / 4$ and $q=1$.

The dynamical equations of the system are $\left(\dot{p}_{n_{1} n_{2}} \equiv \ddot{u}_{n_{1} n_{2}}=-\partial H / \partial u_{n_{1} n_{2}}\right)$ :

$$
\begin{aligned}
& F_{n_{1} n_{2}}(u, s, \varepsilon)=\ddot{u}_{n_{1} n_{2}}+\omega_{n_{1} n_{2}}^{2} u_{n_{1} n_{2}}-3 s u_{n_{1} n_{2}}^{2}+ \\
& +\varepsilon\left(4 u_{n_{1} n_{2}}-u_{n_{1}-1 n_{2}}-u_{n_{1}+1 n_{2}}-u_{n_{1} n_{2}+1}-u_{n_{1} n_{2}-1}\right)=0 .
\end{aligned}
$$

The breathers and Anderson modes solutions are obtained using the same methods as in [5] applied to two-dimensional systems. These are explained in section 3 .

To make the continuation two different samples of breathers (shown in figure 1 and figure 10) are taken at the nonlinear-ordered limit $(s=1)$ and the value of the parameter $s$ is varied until it reaches the linear-disordered limit $(s=0)$. In this path, we keep constant the action $I$ of the system $\ddagger$ and the random vector $r_{n_{1} n_{2}}$ (defined in equation (2)). This is the same procedure used in [5].

\section{Obtaining breathers and Anderson modes}

\subsection{Obtaining breathers}

In order to obtain breathers, we use a variant [5] of the numerical methods described in $[15,16,17]$.

We denote by $\mathcal{E}_{s}^{2}\left(\omega_{\mathrm{b}}\right)$ the space of time-periodic, time-reversible solutions of frequency $\omega_{\mathrm{b}}$, with continuous second derivative. Therefore the functions $u_{n_{1} n_{2}}(t)$ can be approximated by truncated Fourier series of the form:

$$
u_{n_{1} n_{2}}(t)=\sum_{k=-k_{m}}^{k_{m}} z_{n_{1} n_{2}}^{k} e^{\mathrm{i} k \omega_{\mathrm{b}} t} .
$$

The time-symmetry property of $u_{n_{1} n_{2}}(t)$ and the fact that the operator in (4) is real, determine:

$$
u_{n_{1} n_{2}}(t)=z_{0}+\sum_{k=1}^{k=k_{m}} 2 z_{n_{1} n_{2}}^{k} \cos \left(k \omega_{\mathrm{b}} t\right) .
$$

The first step for obtaining a breather is to find the solution for the isolated oscillator with a given frequency $\omega_{\mathrm{b}}$. In order to do this, the Newton-Raphson method is used, being the seed the solution of the harmonic problem.

The second step is to obtain the breather from the anticontinuous limit, which consists of a particle with the previously calculated solution asigned to a particle being the others at rest. This is done by varying with small steps the coupling parameter $\varepsilon$ from $\varepsilon=0$ to the desired value.

$\ddagger$ In this case, the action is the phase-space's area, i.e., $I=\oint p \mathrm{~d} q$ 


\subsection{Anderson modes solutions}

For a harmonic on-site potential, the dynamical equations of the system (Eq. (4)) can be written:

$$
\begin{aligned}
& \ddot{u}_{n_{1} n_{2}}=-\omega(s)_{n_{1} n_{2}}^{2} u_{n_{1} n_{2}}- \\
& -\varepsilon\left(4 u_{n_{1} n_{2}}-u_{n_{1}-1 n_{2}}-u_{n_{1}+1 n_{2}}-u_{n_{1} n_{2}+1}-u_{n_{1} n_{2}-1}\right),
\end{aligned}
$$

or in an abbreviated notation:

$$
\ddot{u}=-\Omega(s)^{2} u \text {. }
$$

The spectrum of $\Omega(s)^{2}$ consists of $m^{2}$ positive eigenvalues, $\tilde{\omega}_{k}(s)^{2}$ which correspond to the square of the phonon frequencies for a particular value of $s$. The eigenvectors of $\Omega(s)^{2}, v^{k}(s)$, are called Anderson modes and are spatially localised (cf. [18]) for our particular disorder configuration. Thus, equation (8) has timereversible, spatially localised, periodic solutions:

$$
u_{n_{1} n_{2}}^{k}(t)=\cos \left(\tilde{\omega}_{k} t\right) v_{n_{1} n_{2}}^{k}
$$

\section{Bifurcation analysis}

We have analyzed a system with Hamiltonian (1), taking $\varepsilon=0.01$ and $m=17$, so that the number of particles is $17 \times 17$, which proves to be large enough to observe LOs. The number of Fourier components of the solution are 10, which may appear small, but truncation has been carried out when the last Fourier coefficient is $\sim 10^{-6}$ times the magnitude of the largest one. Besides, when the degree of linearity increases, this ratio decreases.

The initial seed used in the continuation procedure at $s=1$ is the single breather solution obtained from the uncoupled limit, i.e. $\epsilon=0$, with all the particles at rest except the central one $\left(n_{1}=9, n_{2}=9\right)$, which oscillates with phase 0 at $t=0$ and frequency $\omega_{\mathrm{b}}=0.85$. This allows a relatively large window of values outside the linear modes band [2]. The resulting value of the action is $I=0.1035$, and the profile of this breather is shown in figure 1.

It could be expected that the bifurcations we obtained in the path from the "breather limit to the "Anderson limit were either inverse pitchforks or saddle nodes because an enormous number of solutions must be connected to a relatively small number of them. The number of possible LOs in an square lattice of $m \times m$ particles, at the nonlinear ordered limit (multibreathers) is $\left(9^{m}-1\right) / 2$, while the number of possible LOs at the linear disordered limit (Anderson modes) is only $\mathrm{m}^{2}$.

We present now the results obtained for different paths (3) from the nonlinear ordered case $(s=1)$ to the linear disordered case $(s=1)$ :

\subsection{Path $q=1 / 4$}

The path begins taking at $s=1$ the breather in figure 1 . The continuation finishes at $s=0$ in the Anderson mode shown in figure 2. When $s$ decreases there exists a bifurcation for a value of $s$ in the interval $(0.264,0.265)$. Figure 3 shows the breather profile just before and after this bifurcation.

At the bifurcation point, the LO frequency enters the linearized modes band (figure 4), which enables us to confirm that we have found a bifurcation. 


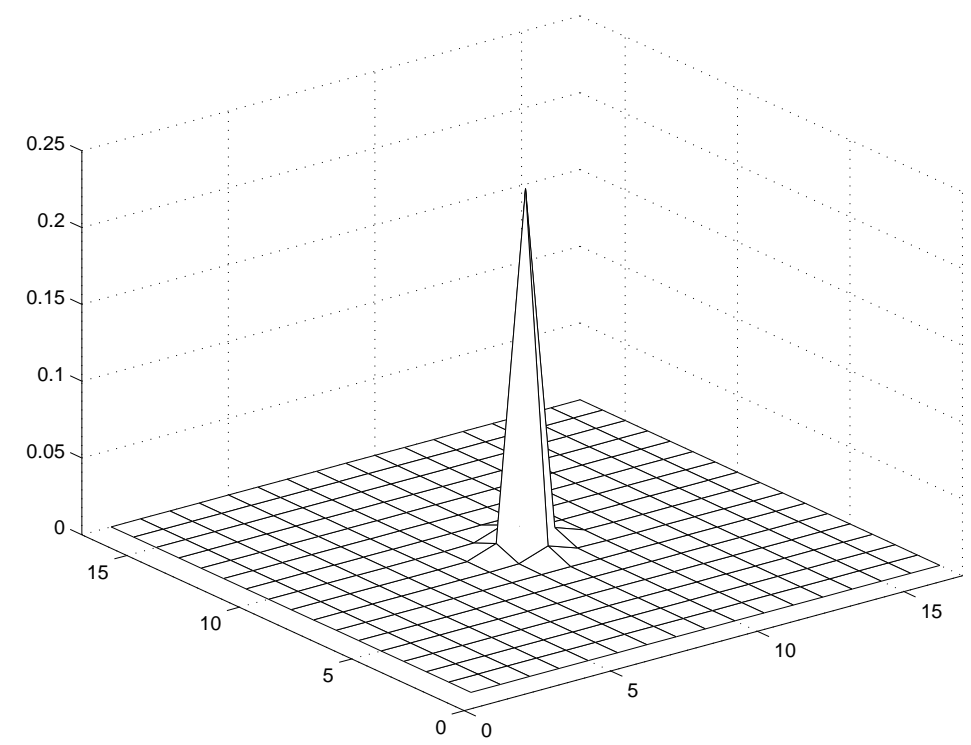

Figure 1. Profile of the breather used as seed for obtaining pitchfork bifurcations $\left(\omega_{b}=0.85\right.$ and $\left.s=1\right)$.

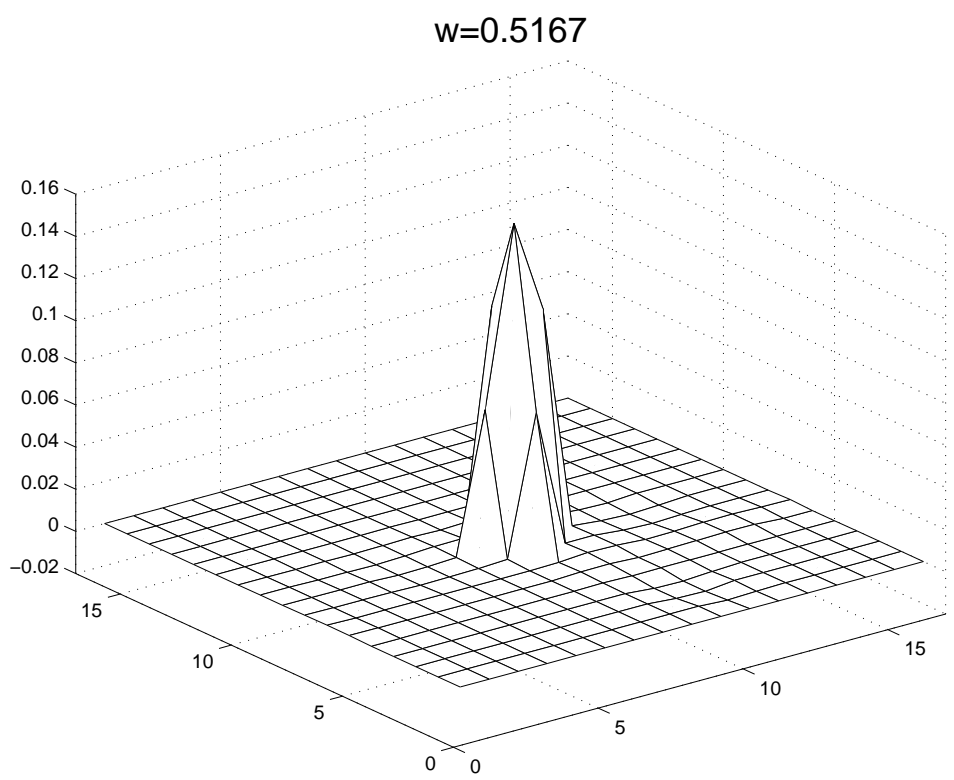

Figure 2. Profile of the Anderson mode which are at the end of the $1 / 4$ path.

The bifurcation is an imperfect pitchfork, which means that the branches are slightly separated, as can be seen in figure 5. The central branch corresponds to the initially continued solution, while the outer branches are obtained adding or subtracting to the solution in a point of the central branch (not very close to the 


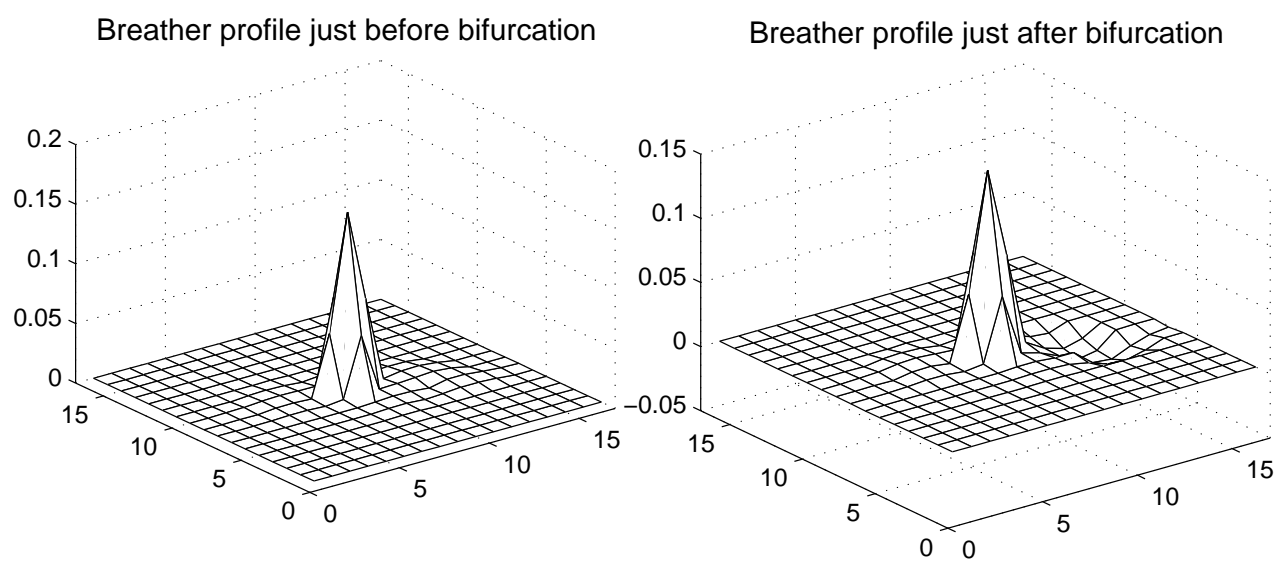

Figure 3. LO before $(s=0.265)$ and after $(s=0.264)$ the bifurcation. $1 / 4$ path.

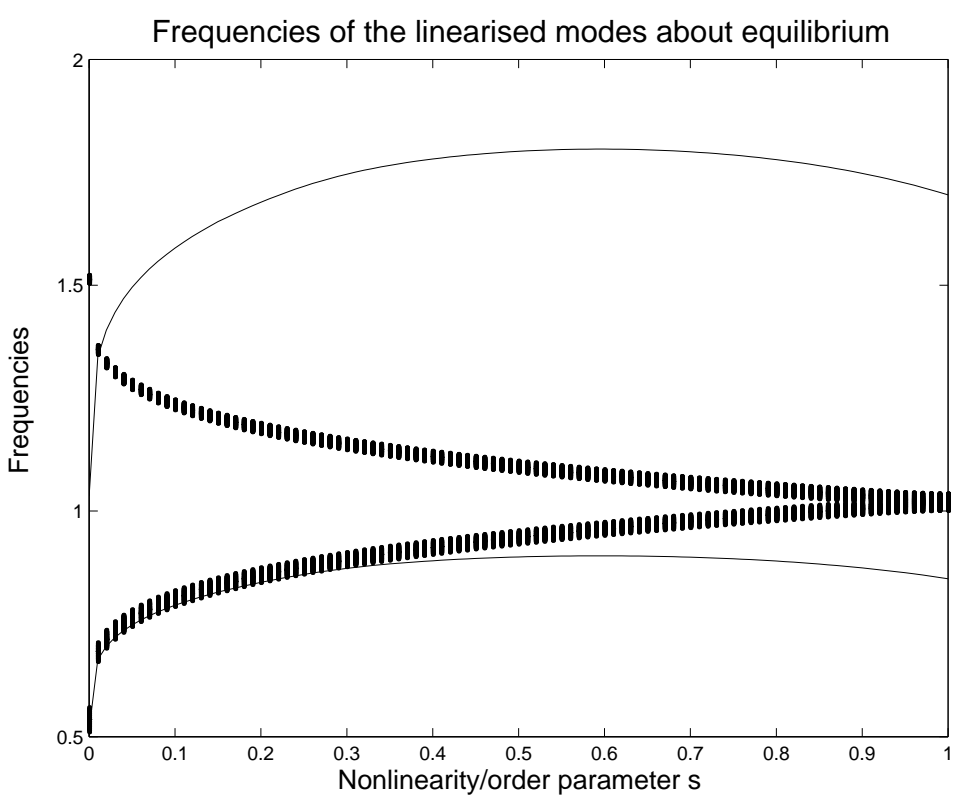

Figure 4. Frequencies of the LOs (also twice its frequency) and all the linearised modes about equilibrium for the $1 / 4$ path. It can be seen how the breather frequency enters the phonon band when the continuation parameter decreases, concretely between $s=0.265$ and $s=0.264$.

bifurcation) the eigenvector corresponding to the centre subspace, and using the new vector as a seed for the Newton method. The LOs at the end of the outer branches are shown in figure 6 . The main difference between the outer branches and the central one is the existence of a "hump" centered in the particle $\left(n_{1}=13, n_{2}=8\right)$.

A linear stability analysis [2] of the solutions shows that all the branches are linearly stable. 


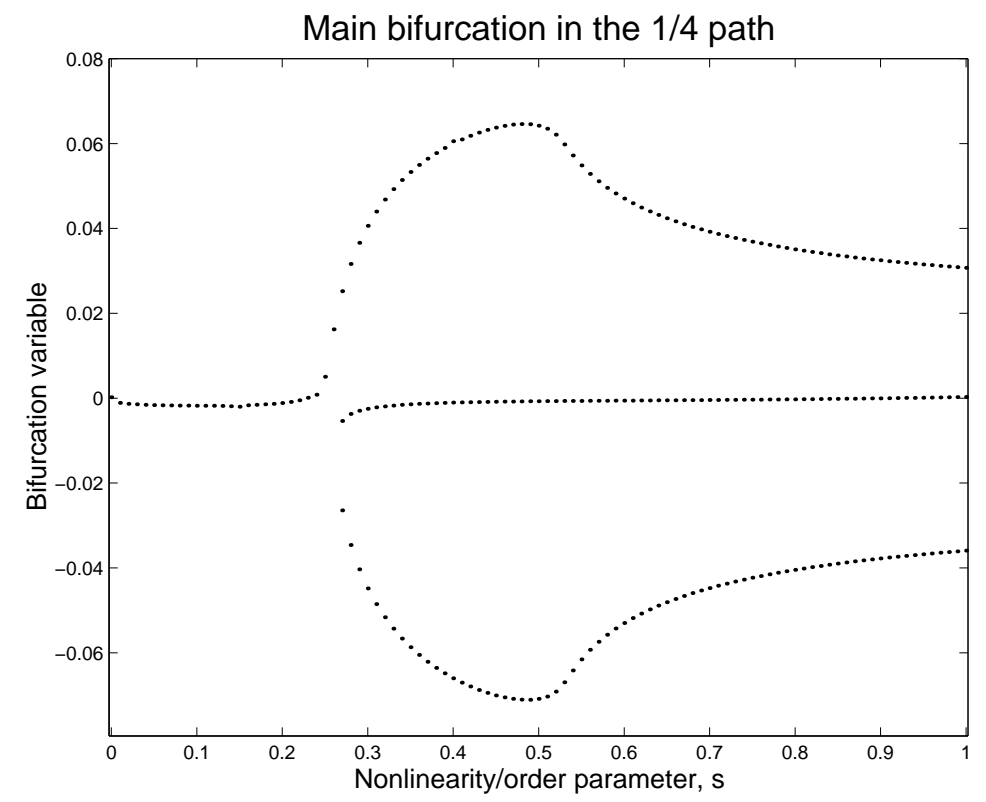

Figure 5. Bifurcation diagram in the $1 / 4$ path.

Breather profile at the upper branch

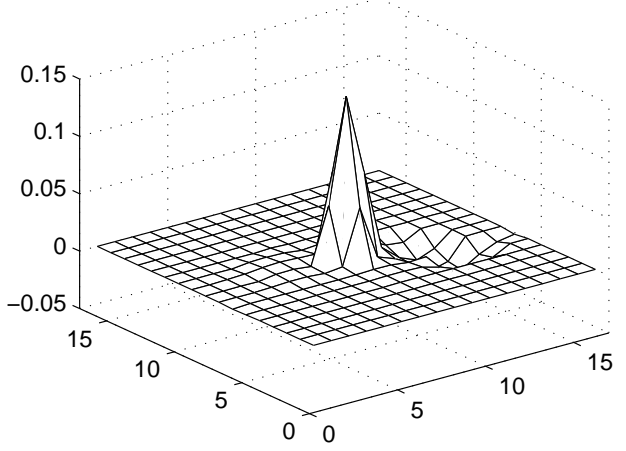

Breather profile at the lower branch

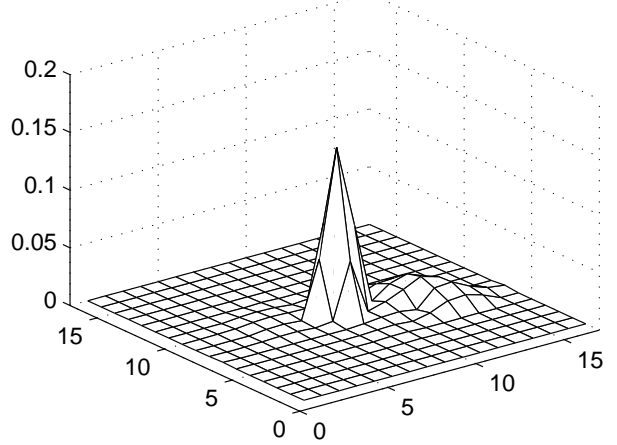

Figure 6. LO just before bifurcation $(s=0.265)$ in the upper and lower branches of the pitchfork. 1/4 path.

\subsection{Linear path}

This path begins taking at $s=1$ the same breather as before (shown in figure 1). The continuation finishes at $s=0$ in the Anderson mode shown in figure 7. When $s$ decreases there exists an inverse period-doubling bifurcation for a values of $s$ within the interval $(0.336,0.337)$. At this value, all the odd Fourier components of the solution become zero.

In figure 8 it is shown the breather profile before the bifurcation, which corresponds to the lower branch in the bifurcation diagram (figure 9). The profile of the upper branch breather is symmetric to the represented in figure 8 , because 


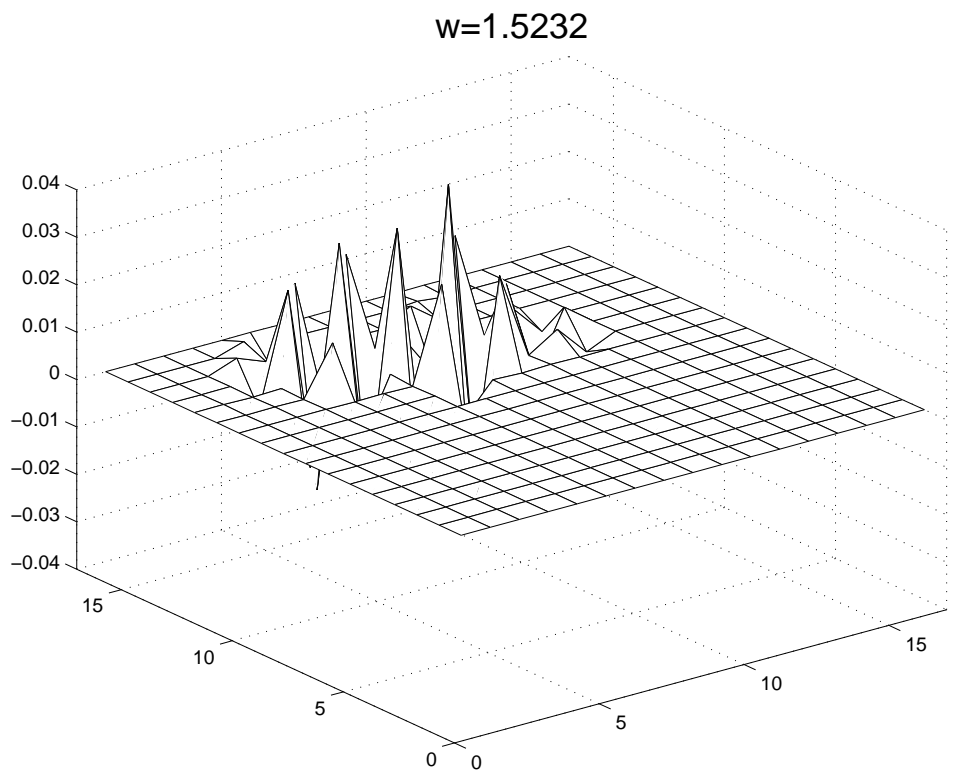

Figure 7. Profile of the Anderson mode which are at the end of the linear path.

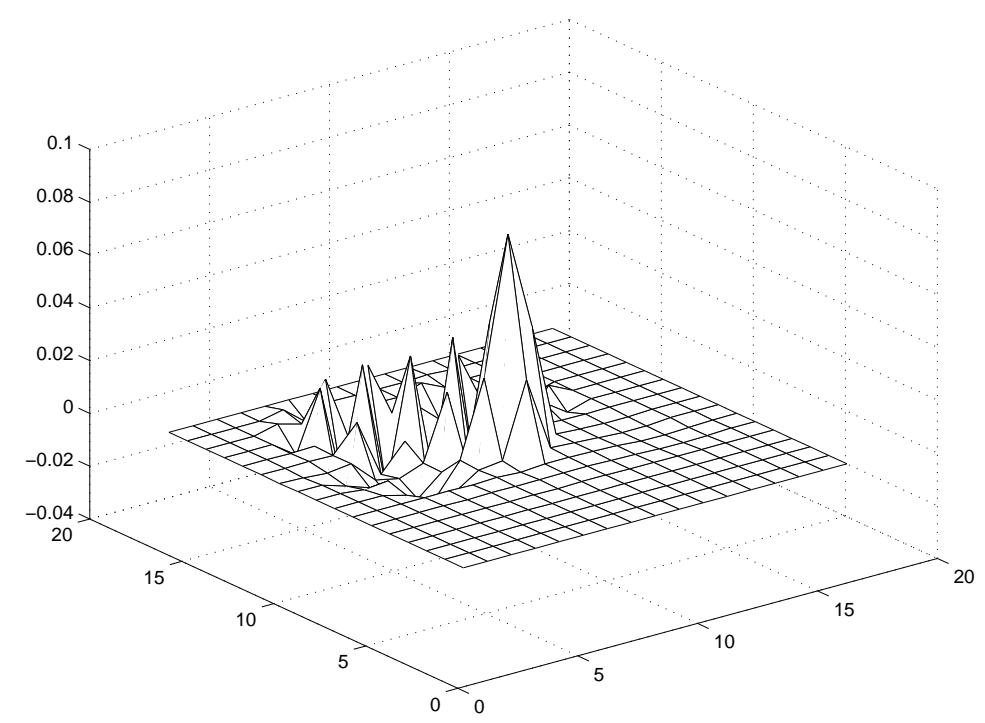

Figure 8. Breather profile just before bifurcation $(s=0.337)$ in the lower branch. The profile in the upper branch is symmetric with respect to the XY-plane. Linear path. 


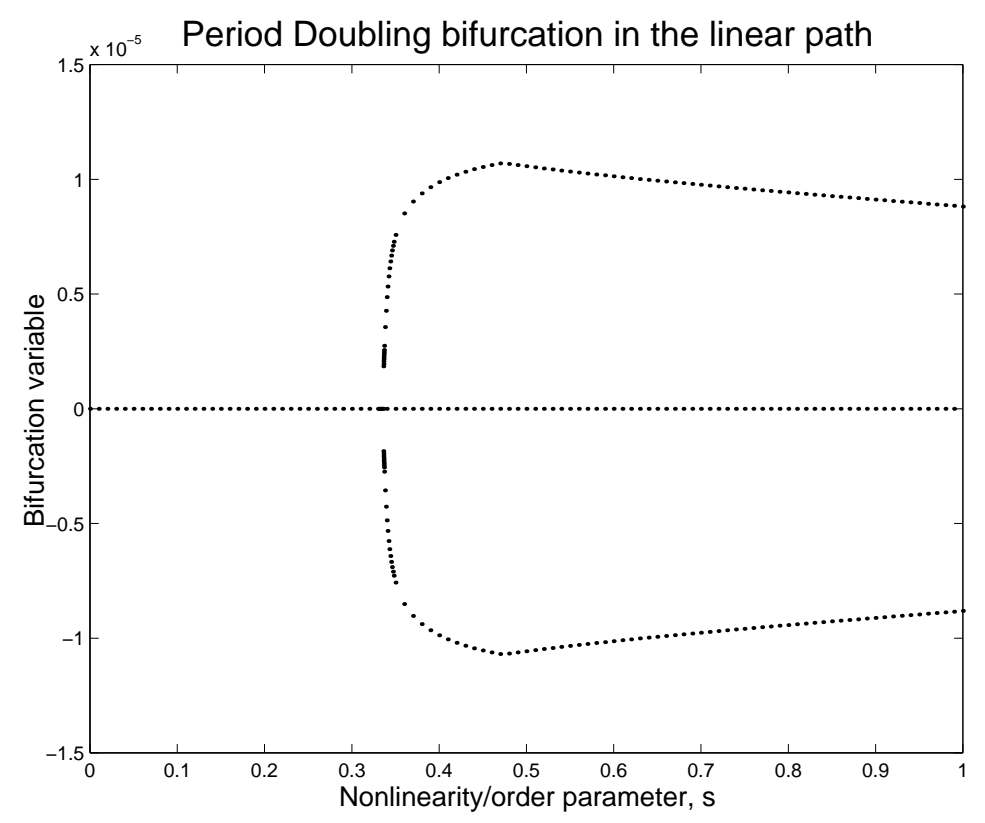

Figure 9. Inverse period-doubling bifurcation in the linear path.

the bifurcation is an inverse period-doubling. The profile of the breather after the bifurcation, i.e. from $s=0.336$ to $s=0$, is similar to the Anderson mode in figure 7. The central branch of the pitchfork bifurcation is LOs which delocalizes when $s$ increases. Numeric calculations show that all these branches are linearly stable.

\subsection{Saddle node in the linear path}

In our system, for all the values of $q$ with the same random vector, and starting with the same one-site breather, a pitchfork bifurcation is obtained. Saddle nodes must appear with the initial breather located in a different site.

Thus, we have chosen as the seed solution at $s=0$ the breather in figure 10. It has the particle $(13,13)$ excited and oscillates with phase 0 at $t=0$ and frequency $\omega_{\mathrm{b}}=0.85$. It corresponds to the lower branch in the bifurcation diagram (figure 11). The breather corresponding to the upper branch has three peaks, in $(13,13),(13,14)$ and $(14,14)$ (figure 10), and oscillates with $\omega_{\mathrm{b}}=0.9701$. The LOs at the lower branch in the bifurcation diagram are linearly stable and the LOs at the upper one are unstable.

The bifurcation occurs at a value of $s$ within the interval $(0.9300,0.9301)$. At this value, the breather has the profile shown in figure 11.

\section{Conclusions}

We have studied the bifurcations that appear in a class of two-dimensional disordered system in the path from nonlinearity-order to linearity-disorder. We have obtained two different types: saddle nodes and inverse pitchforks, either with or without inverse period-doubling. 

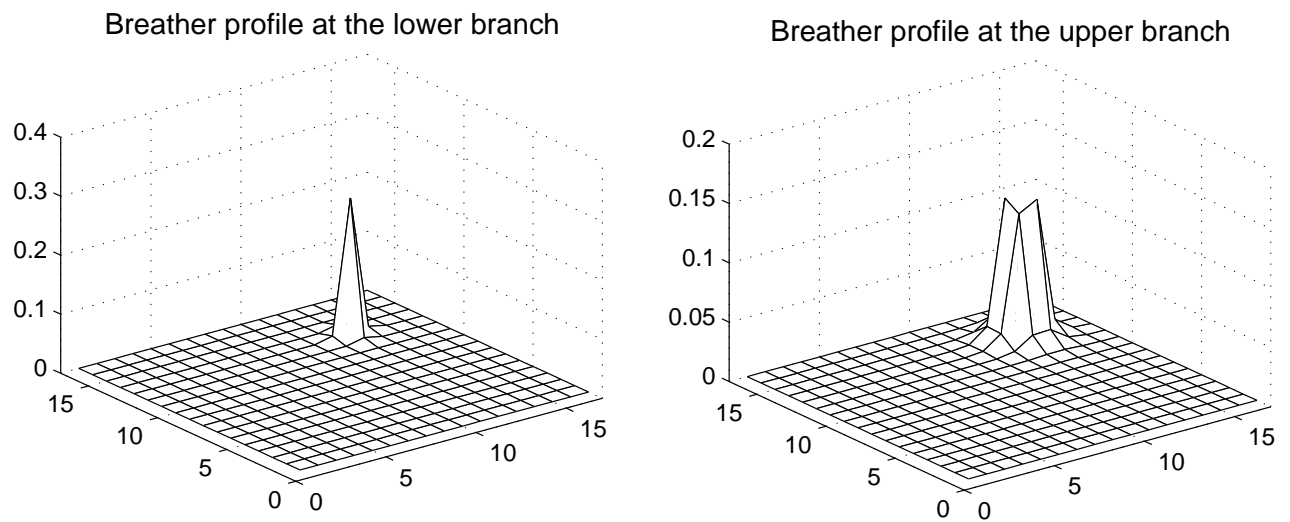

Figure 10. Profile of the breathers corresponding to the upper and lower branch at the turning point in the linear path for $s=1$.

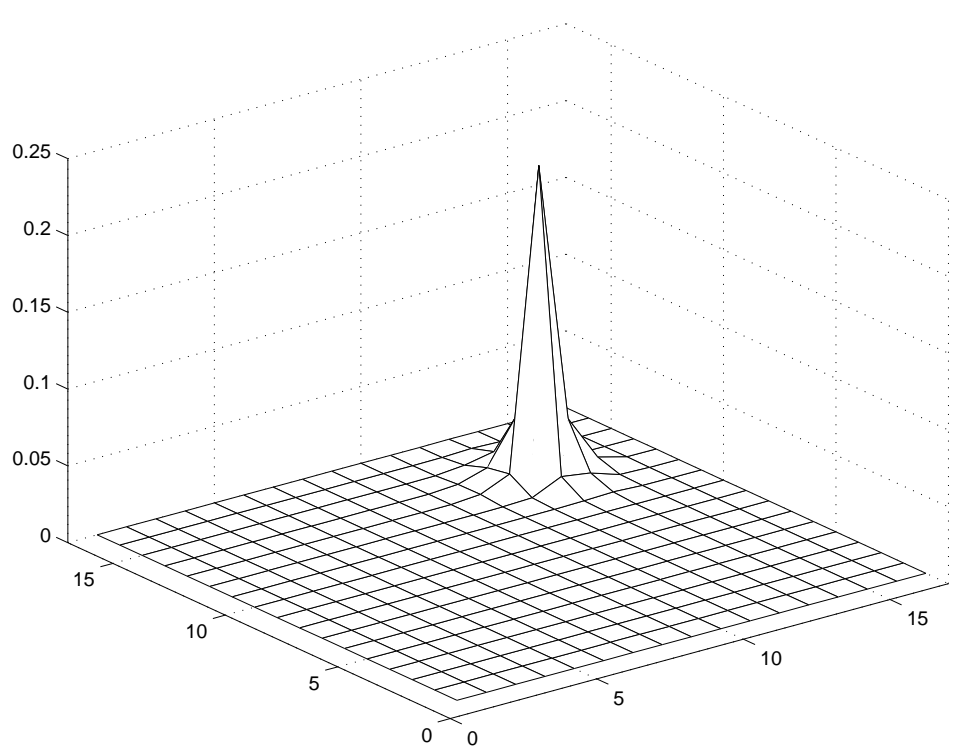

Figure 11. Breather profile at the turning point $(s=0.9301)$. Linear path.

It would be interesting to find out which multibreathers, and in which conditions, can be connected to Anderson modes. This is an impossible numerical task, because in a system with $m=17$, the number of multibreathers is $\sim 10^{16}$.

There are several open questions: Why does appear a period-doubling bifurcation? What is the influence of keeping constant the action? Are these properties maintained by other classes of two-dimensional lattices, that is, with different types of anharmonicity or with random couplings between the particles? The answers need more analytical and numerical study, which will be object of further research. 


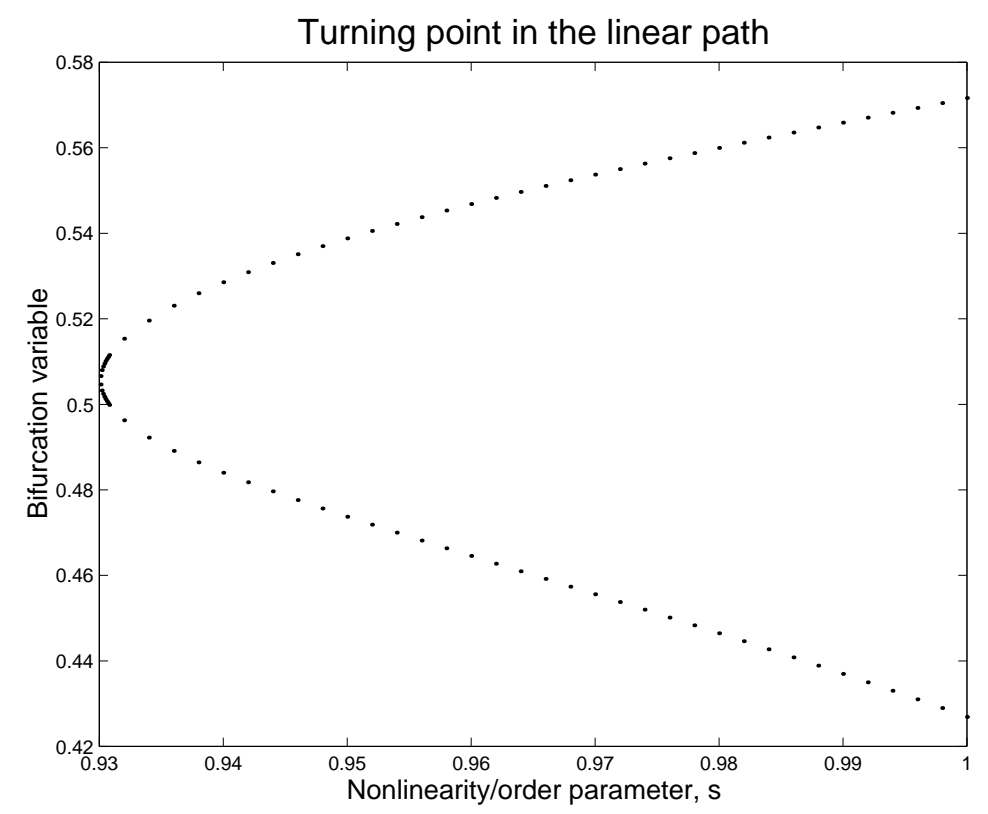

Figure 12. Turning point in the linear path.

\section{Acknowledgments}

This work has been supported by the European Union under the RTN project LOCNET, HPRN-CT-1999-00163

[1] RS MacKay and S Aubry. Proof of existence of breathers for time-reversible or Hamiltonian networks of weakly coupled oscillators. Nonlinearity, 7:1623-1643, 1994.

[2] S Aubry. Breathers in nonlinear lattices: Existence, linear stability and quantization. Physica D, 103:201-250, 1997.

[3] S Flach and CR Willis. Discrete breathers. Physics Reports, 295:181-264, 1998.

[4] PW Anderson. Absence of diffusion in certain random lattices. Phys. Rev., 109:1492-1505, 1958.

[5] JFR Archilla, RS MacKay, and JL Marin. Discrete breathers and anderson modes: two faces of the same phenomenon? Physica D, 134:406-418, 1999.

[6] G Kopidakis and S Aubry. Intraband discrete breathers in disordered nonlinear systems i: Delocalization. Physica D, 130:155-186, 1999.

[7] G Kopidakis and S Aubry. Intraband discrete breathers in disordered nonlinear systems ii: Localization. Physica D, 139(3-4):247-275, 2000.

[8] PG Kevrekidis, KO Rassmussen, and AR Bishop. Two dimensional discrete breathers: construction, stability, and bifurcations. Phys. Rev. E, 61(2):2006-2009, 2000

[9] S Flach, K Kladko, and S Takeno. Acoustic breathers in two-dimensional lattices. Phys. Rev. Lett., 79(24):4848-4841, 1997.

[10] S Flach, K Kladko, and RS MacKay. Energy thresholds for discrete breathers in one-, two-, and three-dimensional lattices. Phys. Rev. Lett., 78(7):1207-1210, 1997.

[11] JL Marín, JC Eilbeck, and FM Russell. Localized moving breathers in a 2-D hexagonal lattice. Phys. Lett. A, 248:225-229, 1998.

[12] JL Marin, JC Eilbeck, and FM Russel. 2-d breathers and applications. In PL Christiansen and MP Soerensen, editors, Nonlinear Science at the dawn of the 21st century, pages 293-306. Springer, 2000.

[13] JL Marin, FM Russell, and JC Eilbeck. Breathers in cuprate superconductor lattice. Submitted for publication, 1999. 
[14] RA Sammut SF Mingaleev, YuS Kivshar. Long-range interaction and nonlinear localized modes in photonic crystal waveguides. Phys. Rev. E, 62(4):5777-5782, 2000.

[15] JL Marin and S Aubry. Breathers in nonlinear lattices: Numerical calculation from the anticontinuous limit. Nonlinearity, 9:1501-1528, 1996.

[16] JL Marin. Intrinsic Localised Modes in Nonlinear Lattices. PhD dissertation, University of Zaragoza, Department of Condensed Matter, June 1997.

[17] JL Marin and S Aubry. Breathers in nonlinear lattices: Numerical methods based on the anti-integrability concept. In L Vázquez, L Streit, and VM Pérez-García, editors, Nonlinear Klein-Gordon and Schrödinger Systems: Theory and Applications, pages 317-323. World Scientific, Singapore and Philadelphia, 1995.

[18] J Frölich, T Spencer, and CE Wayne. Localization in disordered, nonlinear dynamical systems. J. Statist. Phys., 42:247-274, 1986. 